\title{
Factores psicosociales asociados al estrés en profesores universitarios colombianos*
}

\author{
Psychosocial factors related with stress \\ in colombian professors
}

\author{
Mariantonia Lemos* \\ ORCID: 0000-0002-9131-4704 \\ Gabriela Calle \\ ORCID: 0000-0001-8013-1382 \\ Tatiana Roldán \\ ORCID: 0000-0002-6986-921X \\ Melissa Valencia \\ ORCID: 0000-0001-9118-413X \\ Johnny Javier Orejuela \\ ORCID: 0000-0001-9181-463X \\ Juan Pablo Román-Calderón \\ ORCID: 0000-0002-4352-8513 \\ Universidad EAFIT, Colombia \\ Recibido: 27 de marzo de 2018 \\ Revisado: 26 de abril de 2018 \\ Aceptado: 24 de mayo de 2018
}

\section{Resumen}

Objetivo: evaluar los factores psicosociales asociados al estrés en una muestra de profesores de una universidad privada colombiana. Método: Estudio descriptivo correlacional de una muestra intencional de 61 profesores (rango de edad 25 a 63 años; 65,6\% hombres). Se utilizaron el cuestionario de salud del paciente PHQ-9, Cuestionario de Ansiedad generalizada GAD-7, Escala de estrés percibido PSS-14 y un cuestionario sobre factores psicosociales laborales. Adicionalmente se midió la presión arterial. Resultados: El 21,3\% de los profesores presentan niveles significativos de estrés. Hay mayor necesidad de trabajar en casa, interferencia familia-trabajo y trabajo-familia en profesores con estrés, mientras que el control es menor. Cabe anotar que estos profesores también presentan mayor sintomatología emocional. El modelo final mostró que la necesidad de trabajar en casa y la interferencia familia - trabajo explican el 45,6\% de la varianza en el estrés laboral de los profesores.

Palabras clave: factores psicosociales, estrés laboral, trabajo docente, docencia universitaria.

Artículo de investigación. Citar como: Lemos, M., Calle, G., Roldán, T., Valencia, M., Orejuela, J. J., \& Román-Calderón, J. P. (2019). Factores psicosociales asociados al estrés en profesores universitarios colombianos. Diversitas: Perspectivas en Psicología, 15(1), 59-70. DOI: https:// doi.org/10.15332/s1794-9998.2019.0001.05

Autor de correspondencia: Mariantonia Lemos. Profesora titular. Programa de psicología, Escuela de Humanidades, Universidad EAFIT. Cra 49 \# 7 sur 50. Medellín - Colombia. Teléfono: 2619500, ext 8923. Correo electrónico: mlemosh@eafit.edu.co 


\section{Abstract}

Objective: to evaluate the psychosocial factors associated with stress in a sample of professors from a private Colombian university. Method: Correlational descriptive study of an intentional sample of 61 professors (age range 25 to 63 years, 65,6\% men). The patient's health questionnaire PHQ-9, Generalized Anxiety Questionnaire GAD-7, Perceived Stress Scale PSS-14 and a questionnaire of psychosocial work factors were used. In addition, blood pressure measurements were taken. Results: $21,3 \%$ of professors had significant levels of stress. There are more need to work at home, family-work and work-family interference in professors with stress, while control is lower. It should be noted that these teachers also have greater emotional symptoms. The final model showed that the need to work at home and the family - work interference explain $45,6 \%$ of the variance in teachers' work stress.

Keywords: psychosocial factors, work stress, teaching work, university teaching.

\section{Introducción}

El estrés puede definirse como un proceso que se desarrolla en la relación entre la persona y el ambiente, en el cual una situación o condición interna o externa es valorada por la persona como superior a sus recursos (Steptoe \& Ayers, 2004). La valoración que hace el individuo es un elemento fundamental y en ésta influyen tanto los factores individuales como las características propias del estímulo estresor. El tipo de trabajo de una persona, así como los estresores que tiene en el mismo, podrían ser factores adquiridos, tanto moderadores como de riesgo, para las consecuencias del estrés en la salud (Lazarus, 1999; Lazarus \& Folkman, 1986).

La evidencia ha mostrado que condiciones laborales como la ambigüedad del rol, el trabajar largas horas o el trabajo excesivo, la variabilidad y la extensión de los turnos laborales, la falta de control del proceso y las condiciones de trabajo, de excesiva o poca variabilidad en el trabajo o de líderes que apoyen, tienen el potencial de afectar la calidad de vida de las personas y de aumentar los niveles de estrés (Dobson \& Schnall, 2011; Gordon \& Schnall, 2011; Kinman \& Jones, 2001; Landsbergis, Schnall, \& Dobson, 2011). Las desbordadas exigencias, los cambios en los horarios y el acoso laboral son factores de riesgo para sufrir trastornos mentales (Marchand, Demers, \& Durand, 2005). Por ejemplo, se ha señalado una relación directa entre tensión laboral, es decir la combinación de altas demandas y bajo control, con depresión (Dobson \& Schnall, 2011; Vieco-Gómez \& Abello-Llanos, 2014). Igualmente, se ha afirmado que en las últimas décadas el bajo apoyo social en el trabajo y el bajo control laboral se han incrementado llevando a un mayor riesgo de morbilidad psiquiátrica. Adicionalmente, se encuentra que las demandas y el poco control se asocian con el burnout, el cual podría ser un mediador para el desarrollo de depresión en personas con altas exigencias y bajo control (Bergman et al., 2012).

Por otra parte, se ha encontrado que el número de roles que debe cumplir una misma persona tiende a ser una fuente de estrés, especialmente cuando las expectativas en uno de ellos obstaculizan el cumplimiento de las expectativas en otros. En este sentido, el rol laboral y la falta de equilibrio o no conciliación en la relación trabajo-vida personal ha mostrado ser una fuente de estrés para los trabajadores (Gomez, 2006; Kinman \& Jones, 2001; Ryff, 2014). No obstante, hay que señalar que los factores psicosociales en el trabajo también pueden tener un impacto positivo en la calidad de vida de los trabajadores. Se ha encontrado que trabajar en el área de estudio, percibir que se tienen oportunidades de desarrollo personal y profesional en el trabajo y que lo que se hace tiene alguna trascendencia social son condiciones favorecedoras de experimentar bienestar psicológico en el trabajo (Montoya, 
Panesso, Orozco, \& Orejuela, 2016; Orejuela \& Ramírez, 2011).

Por otro lado, el ámbito laboral de un profesor universitario implica enfrentarse con demandas que en alguna medida se encuentran en otros roles laborales. Sin embargo, hay particularidades propias del trabajo docente que llevan a que algunos factores psicosociales requieran mayor atención que otros. Adicionalmente, la masiva privatización de la educación y el consecuente cambio en el rol del estudiante de beneficiario a cliente, las condiciones actuales de trabajo precario, así como exigencias ligadas a las nuevas tendencias pedagógicas (por ejemplo, educación virtual) llevan a que el docente deba pasar de ser un instructor únicamente a ser un guía o tutor de los estudiantes. Esto implica para el docente mayor involucramiento con sus estudiantes y habilidades para acompañar a éstos no sólo en el ámbito académico sino también en el personal (Rajak \& Chandra, 2017). El campo docente exige el desarrollo simultaneo de competencias interpersonales, didácticas, investigativas y administrativas (Picazo, Guevara \& Ojeda, 2015).

Estudios con profesores universitarios españoles han señalado que esta población es vulnerable a presentar altas exigencias psicológicas, baja autoestima, exigencias del ambiente familiar y laboral, bajo apoyo social e inseguridad elevada en el empleo (García, Iglesias, Saleta \& Romay, 2016). Así mismo, un estudio llevado a cabo con docentes universitarios colombianos encontró que el conflicto trabajo - familia modera la relación entre la tensión laboral y el agotamiento emocional (Gomez, Perilla-Toro \& Hermosa-Rodriguez, 2015). Por otra parte, también se ha encontrado que el rol de docente universitario se considera por muchos un trabajo con alto control y demandas, lo que se concibe como un trabajo activo, así como con posibilidades de desarrollo y aprendizaje (García et al., 2016; Laca-Arocena, Mejía-Ceballos, Yáñez-Velasco \& Mayoral-Sánchez, 2011).
La importancia de los factores psicosociales se ha venido señalando en la literatura sobre salud ocupacional y psicología laboral hace varios años. Sin embargo, los estudios en Latinoamérica son escasos en proporción con los de otros lugares del mundo, además que en ocasiones éstos se han centrado en las enfermedades ocupacionales y no sobre las condiciones psicosociales laborales (Gomez, JuarezGarcia, Feldman, Blanco \& Verá, 2011), aspecto en el que se centra este artículo. Así, este estudio respondió a la necesidad de realizar una evaluación de los factores psicosociales en profesores universitarios, así como sus niveles de estrés, depresión y ansiedad.

\section{Método}

\section{Tipo de Estudio}

Estudio descriptivo correlacional de corte transversal.

\section{Participantes}

Se evaluó una muestra de 61 profesores de una universidad privada colombiana. Se seleccionó una muestra por conveniencia, mediante una convocatoria pública a participar mediante correo electrónico. La edad promedio fue de 41,4 años (rango de edad 25 a 63 años), el 65,6\% fueron hombres y el $34,4 \%$ mujeres. El $63,9 \%$ de los participantes estaban casados y un $27,4 \%$ solteros. Referente a la formación académica se encontró que un $54,1 \%$ tenían títulos de maestría, un 31,1\% de doctorado y los demás eran profesionales $(14,8 \%)$. Con respecto a la situación laboral, el 37,4\% tenía contrato indefinido y $21,3 \%$ fueron profesores de cátedra. En promedio los profesores dictaban 6,5 horas de cátedra a la semana (tabla 1). 
Tabla 1. Caracterización de la muestra

\begin{tabular}{llcc}
\hline & & $n(\%)$ & $M(D E)$ \\
\hline Edad & & $41,4(10,0)$ \\
\hline Sexo & Hombres & $40(65,6)$ & \\
\hline \multirow{3}{*}{ Estado civil } & Mujeres & $21(34,4)$ & $39(63,9)$ \\
& Casado & $4(6,6)$ & \\
\hline \multirow{2}{*}{ Estrato } & Separado & $17(27,9)$ & $1(1,6)$ \\
\hline \multirow{2}{*}{ Escolaridad } & Soltero & $16(26,2)$ \\
& Viudo & $45(73,8)$ & $9(14,8)$ \\
& Medio & $33(54,1)$ & $19(31,1)$ \\
\multirow{2}{*}{ Estudios actuales } & Alto & $36(59)$ \\
& Profesional & $11(18)$ \\
\hline \multirow{2}{*}{ Tipo de contrato } & Magíster & $14(23)$ & $35(57,4)$ \\
& Doctor & $26(42,6)$ \\
\hline Vinculación & Ninguno & $48(78,7)$ \\
& MSc & $13(21,3)$ \\
\hline
\end{tabular}

M: Media, DE: Desviación estándar

Fuente: elaboración propia.

\section{Instrumentos}

\section{Cuestionario de Salud del Paciente (PHQ 9)}

Cuestionario que evalúa cada uno de los nueve criterios del DSM IV para depresión en una escala que va desde cero (para nada) hasta tres (casi todos los días). Una puntuación total de menos de ocho indica que la persona no tiene depresión, una puntuación total de ocho o nueve indica posible depresión y una puntuación mayor indica probable depresión. La sensibilidad y especificidad de la prueba es del 88\% (Kroenke \& Spitzer, 2002; Spitzer, Kroenke \& Williams, 1999).

\section{Cuestionario de Ansiedad Generalizada (GAD 7)}

Cuestionario para identificar casos de ansiedad generalizada y evaluar la gravedad de los síntomas. La escala original tenía 13 ítems, 9 de estos de los síntomas encontrados en el DSM-IV y 4 de estos de otras escalas de medición de la ansiedad. Para el GAD-7 se tomaron los 7 ítems de mayor correlación, los cuales alcanzaron una sensibilidad del $89 \%$ y una especificidad del $82 \%$. La consistencia interna de la prueba fue de 0,92 (Spitzer, Kroenke, Williams \& Löwe, 2006). 


\section{Escala de estrés percibido (PSS 14)}

Instrumento de autoinforme para evaluar el nivel de estrés percibido durante el último mes. Consta de 14 ítems con un formato de respuesta de una escala de cinco puntos. La puntuación directa obtenida indica que a una mayor puntuación corresponde un mayor nivel de estrés percibido. Su validación en Colombia arrojó un alfa de Cronbach de 0,87 (Campo-Arias, Bustos-Leiton \& Romero-Chaparro, 2009) . En la escala original se plantea que el punto de corte que identifica los niveles significativos de estrés se establece con el valor de la media más una desviación estándar (Cohen, Mermelstein, Kamarck \& Hoberman, 1985), el proceso se llevó a cabo tomando como datos de base las puntuaciones normativas que se tenían en el país (Campo-Arias et al., 2009).

\section{Cuestionario de factores psicosociales en el trabajo}

Preguntas orientadas a indagar la valoración de diferentes factores de riesgo psicosociales en una escala de uno (factor no presente) a diez (factor fuertemente presente).

\section{Procedimiento}

Los profesores fueron invitados a participar mediante una convocatoria abierta. Aquellos que aceptaron participar firmaron el consentimiento informado, completaron el protocolo de evaluación y se les hizo una toma de su presión arterial mediante un tensiómetro digital de brazo, esto posterior a un período de reposo de 10 minutos. Los auxiliares de investigación fueron previamente entrenados para la evaluación. Esta investigación cumplió con todos los requerimientos éticos exigidos para las investigaciones con seres humanos.

\section{Análisis de datos}

Inicialmente se obtuvieron los estadísticos descriptivos para cada una de las variables evaluadas, se obtuvieron las prevalencias de depresión y ansiedad según los criterios de cada una de las pruebas diagnósticas. Así mismo, se categorizó la población de acuerdo con la puntuación en el PSS 14 para identificar la prevalencia de estrés clínicamente significativo. Los niveles de presión arterial fueron categorizados de acuerdo con criterios internacionales (Chobanian et al., 2003). Posteriormente, se llevaron a cabo análisis bivariados mediante correlaciones de Pearson para las variables cuantitativas en relación con el estrés visto como la puntuación total en el PSS-14. Finalmente, se llevó a cabo un análisis multivariado mediante regresión lineal múltiple para evaluar la relación con del estrés con los factores de riesgo psicosociales, controlando por sexo y edad. Todos los análisis se llevaron a cabo en SPSS versión 23.

\section{Resultados}

\section{Estrés, indicadores de salud mental y presión arterial}

De acuerdo a los resultados de las pruebas, el $21,3 \%$ de los profesores presentan niveles significativos de estrés. Así mismo, un 6,6\% de los profesores alcanzaron puntuaciones significativas para depresión y un 9,8\% para ansiedad. Con respecto a la presión arterial el $62,3 \%$ presentó niveles normales en presión arterial sistólica (PAS), un $31,1 \%$ presentaron niveles de prehipertesión y un $6,6 \%$ presentaron niveles de hipertensión. Frente a la presión arterial diastólica (PAD) las cifras fueron similares, con $60,7 \%$ con PAD normal, $32,8 \%$ con prehipertensión en esta presión y un $6,6 \%$ con hipertensión (tabla 2). 
Tabla 2. Niveles de estrés y factores emocionales en profesores universitarios.

\begin{tabular}{|c|c|c|c|}
\hline & $M(D E)$ & Rango & $n(\%)$ \\
\hline \multicolumn{4}{|l|}{ Síntomas emocionales } \\
\hline Estrés (PSS 14) & $20,70(10,90)$ & $0,00-44,00$ & \\
\hline Presencia de estrés clínicamente significativo & & & $13(21,3)$ \\
\hline Ansiedad (GAD 7) & $3,90(3,00)$ & $0,00-19,00$ & \\
\hline Presencia de ansiedad clínicamente significativa & & & $6(9,8)$ \\
\hline Depresión (PHQ 9) & $3,80(3,50)$ & $0,00-14,00$ & \\
\hline Presencia de ansiedad clínicamente significativa & & & $4(6,6)$ \\
\hline \multicolumn{4}{|l|}{ Presión arterial } \\
\hline Presión arterial sistólica & $117,11(12,94)$ & $94-152$ & \\
\hline PAS normal & & & $38(62,3)$ \\
\hline PAS prehipertensión & & & $19(31,1)$ \\
\hline PAS hipertensión & & & $4(6,6)$ \\
\hline Presión arterial diastólica & $76,79(9,29)$ & $56-101$ & \\
\hline PAD normal & & & $37(60,7)$ \\
\hline PAD prehipertensión & & & $20(32,8)$ \\
\hline PAD hipertensión & & & $4(6,6)$ \\
\hline
\end{tabular}

M: Media, DE: Desviación estándar, PAS: Presión arterial sistólica, PAD: Presión arterial diastólica

Fuente: elaboración propia.

El análisis de las correlaciones evidenció relaciones positivas entre las puntuaciones de estrés con las de depresión $(r=0,690 ; p<0,001)$, y ansiedad $(r=0,665 ; p<0,001)$. No se encontraron relaciones significativas entre la presión arterial con el estrés, depresión o ansiedad $(p>0,05)$.

\section{Factores psicosociales en profesores universitarios}

Con respecto a los factores psicosociales asociados, se encontró que hay una tendencia general a percibir el trabajo docente con poca ambigüedad y que no hay gran interferencia familia-trabajo, ni tampoco trabajo-familia, aunque se encontraron mayores puntuaciones en ésta última. Por el contrario, se encontró que en promedio los profesores manifestaron sentir tener control sobre su tiempo, variedad en las tareas, posibilidad de aprender en su labor y niveles de apoyo social adecuados. Los factores psicosociales con puntuaciones moderadas fueron las tareas excesivas, la necesidad de llevar trabajo a casa y la desconexión los fines de semana (tabla 3). 
Tabla 3. Factores psicosociales asociados con el estrés en profesores universitarios.

\begin{tabular}{lcc}
\hline & $M(D E)$ & Rango \\
\hline Ambigüedad en las tareas & $2,98(2,66)$ & $1,00-9,00$ \\
Control en el trabajo & $7,27(2,32)$ & $2,00-10,00$ \\
Variedad en el trabajo & $7,66(1,91)$ & $2,00-10,00$ \\
Tareas excesivas & $5,79(2,74)$ & $1,00-10,00$ \\
Necesidad de llevar trabajo a la casa & $5,74(2,80)$ & $1,00-10,00$ \\
Desconexión los fines de semana & $5,65(2,73)$ & $1,00-10,00$ \\
Posibilidad de aprender en el trabajo & $9,00(1,48)$ & $2,00-10,00$ \\
Apoyo social en el trabajo & $7,05(2,59)$ & $1,00-10,00$ \\
Interferencia trabajo familia & $4,54(2,81)$ & $1,00-10,00$ \\
Interferencia familia trabajo & $3,67(2,33)$ & $1,00-10,00$ \\
\hline
\end{tabular}

Fuente: elaboración propia.

\section{Relaciones entre factores psicosociales laborales con el estrés y la presión arterial}

Para llevar a cabo el análisis de los factores psicosociales relacionados con el estrés, inicialmente se llevó a cabo un análisis de las correlaciones mediante el coeficiente de Pearson, el cual reportó que el estrés se asocia negativamente con los años de docencia $(r=-0,292 ; p<0,05)$, con el control que se tiene de las tareas $(r=-0,293 ; p<0,05)$ y poder desconectarse los fines de semana $(r=-0,272 ; p<0,05)$. Así mismo, existe una relación positiva entre el estrés y la ambigüedad de las tareas realizadas $(r=0,284$; $p<0,05)$, las tareas excesivas $(r=0,338 ; p<0,01)$, tener que trabajar en casa, $(r=0,493 ; p<0,001)$ la interferencia del trabajo en la vida familiar $(r=0,463 ; p<0,001)$, la interferencia de la familia en la vida laboral $(r=0,559 ; p<0,001)$. No se encontraron relaciones significativas entre el apoyo social, la posibilidad de aprender, las recompensas, el apoyo social en el trabajo y la variedad de las tareas con los niveles de estrés en la muestra evaluada.

Posteriormente, se procedió a realizar una regresión lineal múltiple por pasos. En la que en el primer paso se ingresó el sexo y la edad como variables de control. Los resultados mostraron que existía una relación lineal entre el estrés y los factores de riesgo psicosociales evaluados, $\left(F_{(4,59)}=13,000 ; p<0,001\right)$. Se encontró que los factores de interferencia familia - trabajo $(t=4,769, p<0,001)$ y la necesidad de trabajar en casa ( $t=3,797 ; p<0,001)$, explicaban el $13,5 \%$ de la varianza de estrés (tabla 4 ).

Tabla 4. Factores Psicosociales Asociados con Estrés en una Muestra de Profesores Universitarios.

\begin{tabular}{lccc}
\hline \multicolumn{1}{c}{ Variable } & B & Error estándar & Beta \\
\hline Interferencia familia - trabajo & 2,27 & 0,477 & $0,485^{* *}$ \\
Necesidad de llevar trabajo a la casa & 1,44 & 0,380 & $0,392^{* *}$ \\
\hline
\end{tabular}

Nota: $R^{2}=0,49(n=59, p<0,001)$. En el modelo se controló por sexo y edad. ${ }^{*} p<0,05{ }^{* *} p<0,001$.

Fuente: elaboración propia. 


\section{Discusión}

Este estudio tenía como objetivo evaluar los factores psicosociales asociados al estrés en una muestra de profesores una universidad privada colombiana, así como estimar los niveles de estrés significativo, depresión y ansiedad. Al respecto se encontró que en general los profesores manifiestan percibir su función docente como una tarea en la que hay alto control, variedad en las tareas y posibilidad de aprendizaje; así como el desempeño de un rol con bajos niveles de ambigüedad y baja interferencia trabajo - familia. De acuerdo con lo anterior, podría pensarse que la percepción de los profesores universitarios evaluados en este estudio coincide con la dimensión de trabajo activo en la que hay demandas pero también un alto control (Karasek, Baker, Marxer, Ahlbom \& Theorell, 1981). Resultados similares fueron reportados en profesores españoles y mexicanos (García et al., 2016; Laca-Arocena et al., 2011).

Este estudio encontró relaciones entre el nivel de estrés percibido con la percepción de mayor ambigüedad, las tareas excesivas, la necesidad de trabajar en casa y la interferencia familia-trabajo y trabajo-familia. Igualmente, los niveles de estrés se asociaron negativamente con el nivel de control percibido, la posibilidad de desconectarse los fines de semana y los años de docencia.

La necesidad de trabajar en casa y las tareas excesivas han sido señaladas en estudios previos siendo reconocida como un indicador de sobrecarga laboral y de esta manera un potencializador del estrés laboral en profesores universitarios (Kuimova, Uzunboylu, Chen, \& Gerasimchuk, 2016; OteroLópez, Santiago Mariño \& Castro Bolaño, 2008; Orejuela, 2018). Al respecto, se ha afirmado que la función de los docentes implica la realización de actividades que en gran parte no se desarrollan en el aula y en ocasiones en el horario de trabajo sino que lo exceden (Picazo et al., 2015). Esto afectaría otros momentos que se espera que sean dedicados al descanso y a la relajación y llevaría a que los profesores no tengan la posibilidad de realizar otras actividades que les permitan compensar el estrés laboral, aumentando el riesgo de padecer enfermedades asociadas con éste (Lemos, Agudelo \& Ríos, 2015; Sterling, 2004). Igualmente, esto po- dría explicar la relación encontrada entre menores niveles de estrés y la posibilidad de desconectarse los fines de semana, de tal forma que el cuerpo tenga la posibilidad de recuperarse de los niveles de activación necesarios para responder a las tareas durante la semana (Sterling, 2004).

El rol docente puede llevar a que con cierta facilidad se presente un conflicto trabajo-familia y familia-trabajo debido a que los profesores pueden verse en la necesidad y deseo de cumplir con la responsabilidad de ambos roles (DiRenzo, Greenhaus \& Weer, 2011). Ambos conflictos se han encontrado asociado a cansancio emocional (Jensen, 2016; Reichl, Leiter \& Spinath, 2014) y la necesidad de sacrificar otros aspectos importantes para los profesores (Wilton \& Ross, 2017). Cabe anotar que la dirección del conflicto que mostró mayor importancia en el estrés laboral fue la interferencia familia - trabajo. Al respecto se tiene que el profesor constantemente dispone del tiempo que debería dedicar a otras actividades o a su familia para estudiar o realizar funciones asociadas con la docencia (Picazo et al., 2015). Sin embargo, esto puede llegar a ser asumido por ellos como parte de su rol, pues la docencia corresponde a los denominados trabajos vocacionales, donde el trabajo en un fin en sí mismo, se hace con un profundo deseo vocacional, no instrumental, y cumple una función autoexpresiva; por lo cual separarlo de la vida personal se hace más difícil (Boltanski \& Chiapello, 2002; Lemos, Roldán-Rojo, Valencia, Calle-González, Goez \& Román-Calderón, 2018). De esta manera, no generaría tanto estrés la interferencia de estas actividades en la vida familiar, sino que la interferencia de la familia en lo laboral es más negativa que la contraria. No obstante, debe tenerse en cuenta que estudios previos han señalado que profesores universitarios bogotanos que presentaban niveles de burnout evidencian mayores niveles de conflicto de la familia hacia el trabajo (Gomez et al., 2015).

Por otra parte, los factores de ambigüedad y control han sido reconocidos como variables importantes en el estrés de los profesores. Al respecto, Picazo et al. (2015) afirman que el estrés laboral podría definirse como el desequilibrio percibido entre las demandas laborales y la capacidad de control, por lo que tener un alto control evitaría tener un alto 
grado de estrés. El control también cobija el tener la seguridad de contar con los medios físicos, tecnológicos y pedagógicos apropiados para llevar a cabo la labor de forma satisfactoria, de tal forma que la ambigüedad en el trabajo aumentaría los niveles de estrés. Consistentemente, los resultados coinciden con en lo descrito por Gillespie et al. (2001) quienes afirman que la falta de experiencia en la docencia genera estrés en los profesores universitarios, ya que es un rol que requiere conocimiento y habilidades (Gillespie et al., 2001), que son adquiridos efectivamente a lo largo de los años.

Alrededor de una quinta parte de los profesores evaluados $(21,3 \%)$ presentaron niveles de estrés significativos. Esto señala que, aunque la docencia universitaria fue percibida como un trabajo asociado a factores psicosociales adecuados, hay una proporción de la población que requiere ser objeto de intervención y estudio para prevenir trastornos emocionales y enfermedades laborales. Con respecto a la relación entre el estrés laboral y síntomas de ansiedad y depresión, estudios previos han evidenciado estas relaciones en población docente perteneciente a colegios (Gomez \& Moreno, 2010) pero también en otras poblaciones (Bergman et al., 2012; Dobson \& Schnall, 2011; Marchand et al., 2005; Vieco-Gómez \& Abello-Llanos, 2014).

Frente a la presión arterial, los niveles de hipertensión en la muestra fueron del $6.6 \%$. Sin embargo, alrededor del $30 \%$ presentan señales de prehipertensión. Aunque en el estudio no se encontró una relación entre la presión arterial y los niveles de estrés, es importante tener presente que las enfermedades laborales, como la hipertensión, pueden tardarse muchos años en desarrollarse, pero una vez aparecen estos cambios de tipo crónico, revertirlos requiere mucho tiempo y en ocasiones no es posible. Por lo tanto, los resultados de este estudio pueden interpretarse como una señal de alerta para promover intervenciones que disminuyan y modifiquen los factores de riesgo psicosociales para evitar la aparición de enfermedades cardiovasculares.

Este trabajo presenta limitaciones que deben ser señaladas. Los resultados señalan que las personas con mayores niveles de estrés presentan mayor sintomatología emocional y que éste se asocia con factores psicosociales. Sin embargo, por el tamaño de la muestra no es posible confirmar si hay una relación entre los trastornos mentales y los factores psicosociales que esté mediada por los niveles de estrés, aspecto que podría responderse mediante estudios de corte longitudinal y análisis estadísticos más avanzados. Adicionalmente, el tamaño de la muestra no permitió realizar comparaciones teniendo en cuenta ciertas variables de control, lo que se considera que podría alimentar la discusión. La relación entre los factores psicosociales y el estrés se establece de forma teórica debido a la naturaleza transversal del estudio; sin embargo debe tenerse en cuenta que estos factores podrían ser también consecuencia del estrés laboral.

En síntesis, el presente trabajo evidencia como el rol de docente universitario se percibe de forma general como un espacio de aprendizaje y un trabajo activo. No obstante, alrededor de una quinta parte de los profesores universitarios evaluados presentan niveles de estrés significativos. El estrés laboral se asocia con una menor percepción de control, la incapacidad de desconectarse los fines de semana y la necesidad de trabajar en casa, así como la sobrecarga de tareas y la ambigüedad en el rol. Adicionalmente, este estudio encontró que el llevar trabajo a casa y la interferencia que causan los asuntos familiares en el trabajo de los docentes son los dos factores de mayor peso en los niveles de estrés percibido, controlando por sexo y edad. Finalmente, se encontró una asociación entre el estrés laboral con sintomatología depresiva y ansiosa.

\section{Referencias}

Bergman, P. N., Ahlberg, G., Johansson, G., Stoetzer, U., Åborg, C., Hallsten, L., \& Lundberg, I. (2012). Do job demands and job control affect problem-solving? Work, 42(2), 195-203. Doi: https://doi.org/10.3233/WOR-2012-1340

Boltanski, L. \& Chiapello, E. (2002). El nuevo espíritu del capitalismo. Madrid: Akal.

Campo-Arias, A., Bustos-Leiton, G. J., \& RomeroChaparro, A. (2009). Consistencia interna y dimensionalidad de la Escala de Estrés Percibido (EEP-10 y EEP-14) en una muestra de 
universitarias de Bogotá, Colombia. Aquichan, 9(3), 271-280.

Chobanian, A. V., Bakris, G. L., Black, H. R., Cushman, W. C., Green, L. A., Izzo Jr, J. L., . . . Wright Jr, J. T. (2003). The seventh report of the joint national committee on prevention, detection, evaluation, and treatment of high blood pressure: the JNC 7 report. JAMA: The Journal of the American Medical Association, 289(19), 2560-2571. Doi: https:// doi.org/10.1001/jama.289.19.2560

Cohen, S., Mermelstein, R., Kamarck, T., \& Hoberman, H. M. (1985). Measuring the Functional Components of Social Support. In I. Sarason \& B. Sarason (Eds.), Social Support: Theory, Research and Applications (Vol. 24, pp. 73-94): Springer Netherlands. Doi: https:// doi.org/10.1007/978-94-009-5115-0_5

DiRenzo, M. S., Greenhaus, J. H., \& Weer, C. H. (2011). Job level, demands, and resources as antecedents of work-family conflict. Journal of Vocational Behavior, 78(2), 305-314. Doi: https://doi.org/10.1016/j.jvb.2010.10.002

Dobson, M. \& Schnall, P. (2011). Del estrés al malestar: el impacto del trabajo en la salud mental. In P. Schnall, M. Dobson, \& E. Rosskam (Eds.), Trabajo No Saludable. Bogota: Uniandes.

García, M. M., Iglesias, S., Saleta, M., \& Romay, J. (2016). Riesgos psicosociales en el profesorado de ense-anza universitaria: diagnóstico y prevención. Revista de Psicología del Trabajo y de las Organizaciones, 32(3), 173-182. Doi: https:// doi.org/10.1016/j.rpto.2016.07.001

Gillespie, N. A., Walsh, M., Winefield, A. H., Dua, J., \& Stough, C. (2001). Occupational stress in universities: Staff perceptions of the causes, consequences and moderators of stress. Work \& stress, 15(1), 53-72. doi: https://doi. org/10.1080/02678370117944

Gomez, V. (2006). Quality of Work and Family Roles and its Relationship with Health Indicators in men and Women. Sex Roles, 55(11-12), 787-799. Doi:https://doi.org/10.1007/s11199-006-9132-x
Gomez, V., Juarez-Garcia, A., Feldman, L., Blanco, G., \& Verá, A. (2011). Por qué una traducción al español de este libro? Aportes para una caracterización de los factores psicosociales en Latinoamérica. In P. Schnall, M. Dobson, \& E. Rosskam (Eds.), Trabajo no saludable. Bogota: Uniandes.

Gomez, V. \& Moreno, L. (2010). Factores psicosociales del trabajo (demanda-control y desbalance esfuerzo-recompensa), salud mental y tensión arterial: un estudio con maestros escolares en Bogotá, Colombia. Universitas Psychologica, 9(2), 393-407.

Gomez, V., Perilla-Toro, L. E., \& HermosaRodriguez, A. (2015). Moderación de la Relación Entre Tensión Laboral y Malestar de Profesores Universitarios: Papel del Conflicto y la Facilitación Entre el Trabajo y la Familia. Revista Colombiana de Psicología, 24(1), 185-201. Doi: https://doi.org/10.15446/ rcp.v24n1.42081

Gordon, D. R. \& Schnall, P. L. (2011). Más allá de lo individual: conexión entre el ambiente de trabajo y la salud. In V. Gomez, L. M. Bonilla, \& T. Laudato (Eds.), Trabajo no saludable: causas, consecuencias, curas (pp. 1-22). Bogotá: Uniandes.

Jensen, M. T. (2016). A two wave cross-lagged study of work-role conflict, work-family conflict and emotional exhaustion. Scandinavian journal of psychology, 57(6), 591-600. doi:https://doi.org/ 10.1111/sjop.12328

Karasek, R., Baker, D., Marxer, F., Ahlbom, A., \& Theorell, T. (1981). Job Decision Latitude, Job Demands, and Cardiovascular Disease. A Prospective Study of Sewdish Men. American Journal of Public Health, 71(7), 694-705. Doi: https://doi.org/10.2105/AJPH.71.7.694

Kinman, G. \& Jones, F. (2001). The Work-home Interface. In F. Jones \& J. Bright (Eds.), Stress. Myth, Theory and Research. Harlow, UK: Pearson Education. 
Kroenke, K. \& Spitzer, R. L. (2002). The PHQ-9: a new depression diagnostic and severity measure. Psychiatric Annals, 32(9), 509-515. Doi: https:// doi.org/10.3928/0048-5713-20020901-06

Kuimova, M. V., Uzunboylu, H., Chen, A. S. M., \& Gerasimchuk, E. V. (2016). Emotional burnout in professional activity of a technical university teacher. Ponte, 72(6), 4. Doi: https://doi. org/10.18844/cerj.v5i2.237

Laca-Arocena, F. A., Mejía-Ceballos, J. C., YáñezVelasco, C. L., \& Mayoral-Sánchez, E. C. (2011). Factores afectivos y de personalidad como antecedentes del bienestar laboral en profesores españoles y mexicanos. Pensamiento Psicológico, 9(16), 11-26.

Landsbergis, P. A., Schnall, P. L., \& Dobson, M. (2011). El lugar de trabajo y las enfermedades cardiovasculares. In P. Schnall, M. Dobson, \& E. Rosskam (Eds.), Trabajo no saludable: causas, consecuencias, curas (pp. 129-159). Bogotá: Uniandes.

Lazarus, R. (1999). Stress and Emotion. A New Synthesis. New York: Springer.

Lazarus, R. \& Folkman, S. (1986). Estrés y procesos cognitivos. Barcelona: Martínez Roca.

Lemos, M., Agudelo, D. M., \& Ríos, P. C. (2015). Reflexiones en torno al estrés y su relación con la enfermedad cardiovascular. Cuadernos Hispanoamericanos de Psicología, 15(2), 5-22.

Lemos, M., Roldán-Rojo, T., Valencia, M., CalleGonzález, G., Goez, I., \& Román-Calderón, J.P. (2018). Overcommitment as mediator between effort and stress in faculty professors. International Journal of Behavioral Medicine, 25(suppl 1), s165. Doi: https://doi.org/10.1007/ s12529-018-9740-1

Marchand, A., Demers, A., \& Durand, P. (2005). Does work really cause distress? The contribution of occupational structure and work organization to the experience of psychological distress. Social science \& medicine, 61(1), 1-14. Doi: https://doi. org $/ 10.1016 / j$.socscimed.2004.11.037
Montoya, F., Panesso, A., Orozco, O., \& Orejuela, J. (2016). Estrategias de afrontamiento en trabajadores con exposicion a factores psicosociales: una aproximacion a su conceptualizacion. En : Orejuela, J., Andrade; V., \& Villamizar, M.(Eds.). Psicologia de las organizaciones y del trabajo: apuestas de investigacion. (pp. 437458). Cali: Bonaventuriana.

Orejuela, J. \& Ramírez, L. A. (2011). Aproximación cualitativa al estudio de la subjetividad laboral en profesionales colombianos. Pensamiento psicológico, 9(16), 125-144.

Orejuela, J. (2018). Clínica del trabajo: el malestar subjetivo derivado de la fragmentación laboral. Bogota: San Pablo-Eafit.

Otero-López, J. M., Santiago Mariño, M. J., \& Castro Bolaño, C. (2008). An integrating approach to the study of burnout in university professors. Psicothema, 20(4), 766-772.

Picazo, D., Guevara, R. M., \& Ojeda, S. L. (2015). Organización del trabajo y exigencias psicosociales en el profesor universitario. Tecnociencia, 9(1).

Rajak, R. \& Chandra, B. (2017). Exploring Predictors of Burnout and Work Engagement among Teachers-A Review on Higher Educational Institutions of India. Journal of the Indian Academy of Applied Psychology, 43(1), 145-156.

Reichl, C., Leiter, M. P., \& Spinath, F. M. (2014). Work-nonwork conflict and burnout: A metaanalysis. Human Relations, 1-27. Doi: https:// doi.org/10.1177/0018726713509857

Ryff, C. D. (2014). Psychological Well-Being Revisited: Advances in the Science and Practice of Eudaimonia. Psychotherapy and psychosomatics, 83(1), 10-28. Doi: https://doi. org $/ 10.1159 / 000353263$

Spitzer, R. L., Kroenke, K., \& Williams, J. B. W. (1999). Validation and utility of a self-report version of PRIME-MD. JAMA: The Journal of the American Medical Association, 282(18), 
1737-1744. Doi: https://doi.org/10.1001/ jama.282.18.1737

Spitzer, R. L., Kroenke, K., Williams, J. B. W., \& Löwe, B. (2006). A brief measure for assessing generalized anxiety disorder: the GAD-7. Archives of Internal Medicine, 166(10), 1092-1097. Doi: https://doi.org/10.1001/archinte.166.10.1092

Steptoe, A. \& Ayers, S. (2004). Stress, Health and Illness. In S. Sutton, A. Baum, \& M. Johnston (Eds.), The SAGE handbook of Health Psychology (pp. 169-198). Londres: SAGE Publications.

Sterling, P. (2004). Principles of allostasis: optimal design, predictive regulation, pathophysiology and rational therapeutics. In J. Schulkin
(Ed.), Allostasis, Homeostasis, and the Cost of Adaptation (pp. 17-64). Cambridge: Cambridge University Press. Doi: https://doi.org/10.1017/ CBO9781316257081.004

Vieco-Gómez, G. F. \& Abello-Llanos, R. (2014). Factores psicosociales de origen laboral, estrés y morbilidad en el mundo. Psicología desde el Caribe, 31(2), 354-385.

Wilton, S. \& Ross, L. (2017). Flexibility, Sacrifice and Insecurity: A Canadian Study Assessing the Challenges of Balancing Work and Family in Academia. Journal of Feminist Family Therapy, 29(1-2), 66-87. Doi: https://doi.org/10.1080/089 52833.2016.1272663 